\title{
Flexible GMR Sensor Array for Magnetic Flux Leakage Testing of Steel Track Ropes
}

\author{
W. Sharatchandra Singh, B. P. C. Rao, S. Thirunavukkarasu, and T. Jayakumar
}

NDE Division, Indira Gandhi Centre for Atomic Research, Kalpakkam 603 102, India

Correspondence should be addressed to B. P. C. Rao, bpcrao@igcar.gov.in

Received 19 November 2011; Accepted 27 December 2011

Academic Editor: Gui Yun Tian

Copyright (C) 2012 W. Sharatchandra Singh et al. This is an open access article distributed under the Creative Commons Attribution License, which permits unrestricted use, distribution, and reproduction in any medium, provided the original work is properly cited.

This paper presents design and development of a flexible GMR sensor array for nondestructive detection of service-induced defects on the outer surface of $64 \mathrm{~mm}$ diameter steel track rope. The number of GMR elements and their locations within saddle-type magnetizing coils are optimized using a three dimensional finite element model. The performance of the sensor array has been evaluated by measuring the axial component of leakage flux from localized flaw (LF) and loss of metallic cross-sectional area (LMA) type defects introduced on the track rope. Studies reveal that the GMR sensor array can reliably detect both LF and LMA type defects in the track rope. The sensor array has a fast detection speed along the length of the track rope and does not require circumferential scanning. It is also possible to image defects using the array sensor for obtaining their spatial information.

\section{Introduction}

Track ropes are a type of wire ropes used for transportation of coal in mining industries. One such rope system is operated for about 10 hours every day to transport 3000 tons of coal with the help of 256 numbers of buckets, each carrying nearly 1.6 tons of coal. The track rope is stationary and is rigidly supported by towers at periodic intervals. As shown in Figure 1, the track rope has 8 layers of stranded wires of different diameters. The 6 inner layers are round-type wires, while the outer two layers are Z-type wires. The round wires are locked by two $Z$ wires to get the strength of the rope. The width of the outer surface of the first $Z$ wire is $6.45 \mathrm{~mm}$, and the gap width between two outer $Z$ wires is $0.76 \mathrm{~mm}$.

During the operation of the rope system, the carriage wheels of the bucket come in contact with the top surface of the outer $\mathrm{Z}$ wire as shown in Figure 2(a). Prolonged use of the rope system is expected to cause abrasion and wear, resulting in loss of metallic cross-sectional area (LMA) or localized flaw (LF) type defects (Figure 2(b)). Also wire breakage and formation of fatigue cracks, pitting corrosion, inter strand nicking or martensitic embrittlement, and so forth are likely to occur $[1,2]$. When more than two $\mathrm{Z}$ wires of the outer layer are broken, they will be separated from the adjacent layers. Detection of damage in track rope is essential as part of the condition monitoring and life management programs. Nondestructive detection of damage in the track rope is challenging due to heterogeneous structure of the rope, multiplicity and uncertainty of broken wires, and hostile working environment.

Among various nondestructive evaluation (NDE) techniques, visual and magnetic flux leakage (MFL) techniques are widely used for monitoring the health of steel track ropes $[3,4]$. Although visual inspection is simple and does not require special instrumentation, it is not suited for monitoring the internal deterioration in track ropes. On the contrary, the MFL technique is capable of detecting both LF and LMA type defects in wire ropes [4].

In MFL technique, wire ropes are locally magnetized using electromagnets or permanent magnets. If any defect is present in the rope, the magnetic flux produced in the rope takes a longer path around the defect and as a result, some amount of flux lines leak out of the surface. Measurement of this leakage flux using sensors forms an important step in MFL testing. The leakage flux has axial (along the rope length) and radial components which can be detected using 


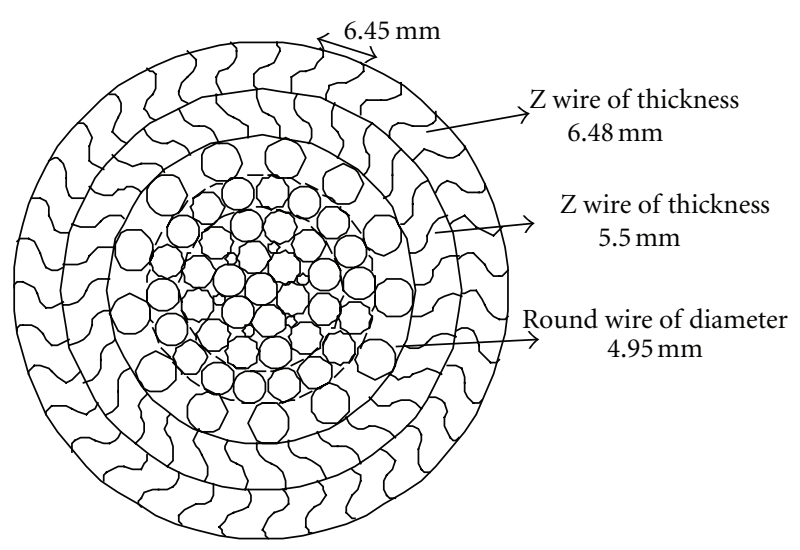

FIGURE 1: The cross-section of double locked track rope.

coils $[5,6]$ or sensors [7-9] and correlated to the size and location of the defects. The magnetization can be continuous $[10]$ or pulsed $[11,12]$. Recently, authors proposed GMR sensor $[13,14]$-based technique for NDE of track ropes, and this requires the use of sophisticated scanning set-up for circumferential scanning over the ropes. Use of array sensors is attractive, and this enables fast and reliable inspection of track ropes [15]. This paper discusses the design and development of a flexible GMR sensor array for detection of damage on the outer surface of the track rope. The performance of the array sensor has been evaluated for fast inspection of track rope and imaging of LF and LMA type defects on the outer Z-wire of the track rope.

\section{GMR Sensor}

GMR sensor consists of a few nm thick multilayer structures $(\mathrm{Co} / \mathrm{Cu} / \mathrm{Co})$ in which ferromagnetic layers are separated by nonmagnetic layers. The sensor works on GMR effect in which there is a large drop in electrical resistance of multilayer for an incident magnetic field, due to the spin dependent scattering of electrons [16]. The GMR sensors are characterized by high sensitivity at low magnetic field and high spatial resolution. They are inexpensive and consume less power [17]. In this study, the GMR sensors are connected in bridge configuration to measure the differential output with high stability. The sensor is encapsulated with standard SOIC- 8 package of $5.9 \times 4.9 \times 1.4 \mathrm{~mm}^{3}$ size. The maximum hysteresis of the GMR sensor is $2 \%$ unit.

\section{Three Dimensional Finite Element Modeling}

In order to identify the number of GMR elements required to cover the top surface of the track rope and to determine the sensor locations, 3D finite element modeling has been performed using COMSOL 3.4 Multiphysics software package. Figure 3(a) shows the mesh generated for the geometry which consists of a track rope (length $300.0 \mathrm{~mm}$, outer diameter $64.0 \mathrm{~mm}$ ) and magnetizing coils. Equation (1) has been solved in three dimensions using the finite element method:

$$
\nabla \times \frac{1}{\mu_{0} \mu_{r}} \nabla \times A=J,
$$

where $A$ is the magnetic vector potential, $\mu_{0}$ is the magnetic permeability of free space, $\mu_{r}$ is the relative permeability, and $J$ is current density. Two saddle coils (length $120.0 \mathrm{~mm}$, width $35.0 \mathrm{~mm}$ ) each consisting of 90 turns with a cross-sectional area of $20.0 \times 10.0 \mathrm{~mm}^{2}$ are used for magnetization of the track rope at a current of $5 \mathrm{~A}$, as shown in Figure 3(a). The magnetizing current in the saddle coils is set in opposite directions to ensure axial magnetization of the rope region between the saddle coils.

For simplicity, in the model the track rope is assumed as a solid rod, and GMR sensor as well as velocity effects is not modeled. The relative magnetic permeability of the rope is assumed constant as 100. Magnetic insulation $(n \times A=$ 0 ) boundary condition is applied at the outer boundaries constructed for the model. The computation time for solving (1) with 5673392 degrees of freedom is approximately 50 minutes in a dual-core 64 bit processor workstation with 8 GB primary memory.

The magnetic vector potential is computed in the solution region, and the axial component of the magnetic flux density $\left(\mathrm{B}_{z}\right)$ between the two saddle coils is predicted. As can be seen from Figure 3(b), the magnetic flux density is nearly uniform for an optimum circumferential intercoil distance of $80.0 \mathrm{~mm}$ that completely covers the expected damage region on the top surface of the Z-wire (dotted region in Figure $3(\mathrm{~b})$ ). This region can accommodate 12 GMR sensors. Hence, a flexible array of 12 GMR sensors has been fabricated and used for detection of damage on the track rope.

\section{Design of GMR Sensor Array}

The layout of the flexible GMR sensor array and its photograph are shown in Figures 4(a) and 4(b), respectively. Each sensor element in the array has a common power input of $5 \mathrm{~V}$, and the array has 12 differential outputs. The overall size of the sensor array is $100 \times 12 \mathrm{~mm}^{2}$ with a centreto-centre distance (pitch) between two sensors of $6.6 \mathrm{~mm}$. The sensor array is kept at the middle of the magnetizing coils. The GMR sensors measure the axial component (along the scan direction) of leakage flux from defects. The array sensor maintains a constant lift-off of $1 \mathrm{~mm}$. The sensors' outputs are acquired and analysed using a LabVIEW-based data acquisition system incorporating averaging and lowpass filter to minimize noise.

\section{Performance Evaluation}

The performance of the sensor array has been evaluated by measuring the axial component of leakage fields from two LF and two LMA type defects in track rope. The two LFs are simulated by electro discharge machining $(\mathrm{EDM})$ notches of size $5.5 \times 2.0 \times 2.0 \mathrm{~mm}^{3}$ (length $\times$ width $\times$ depth) oriented 


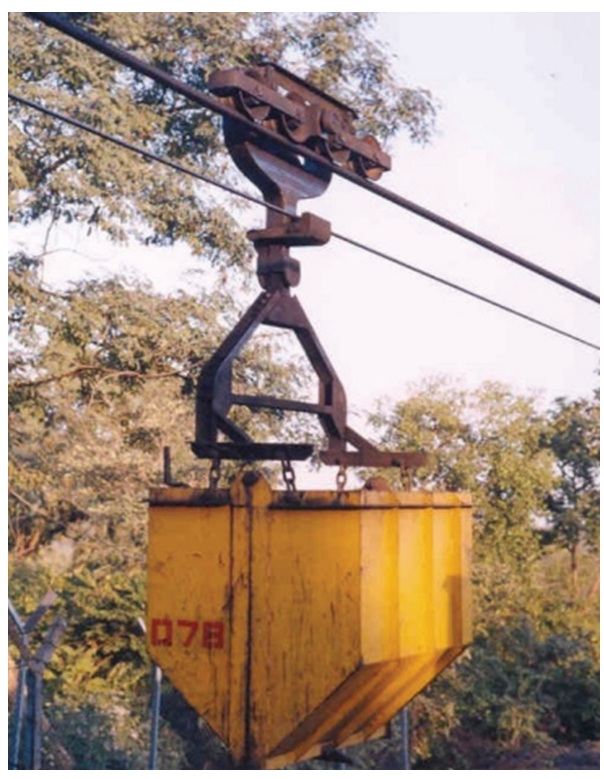

(a)

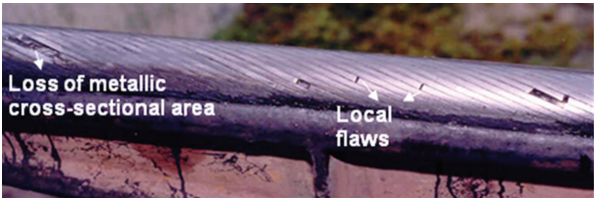

(b)

FIGURE 2: (a) photograph of the track rope system with bucket carrying coal and (b) local flaws and loss of metallic area at the outer surface of the track rope.

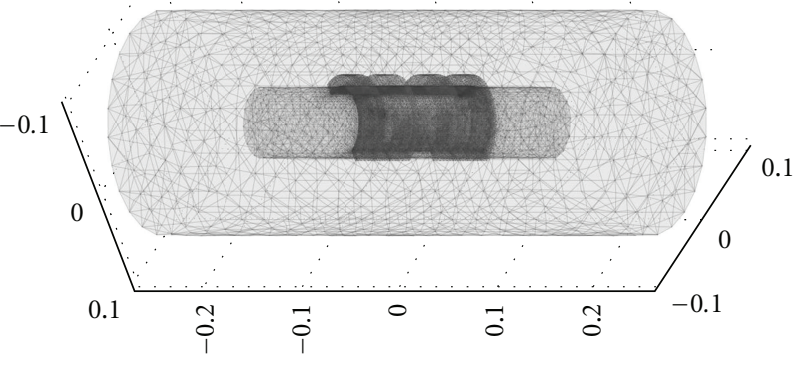

(a)

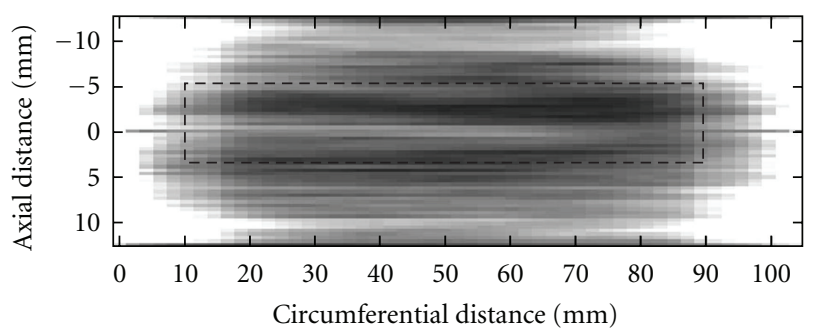

(b)

Figure 3: (a) 3D finite element mesh and (b) predicted magnetic flux density between the saddle coils along half of circumferential distance.

along axial and circumferential directions in the track rope, as shown in Figures 5(a) and 5(b). The two LMAs of sizes $42.0 \times 9.0 \times 3.0 \mathrm{~mm}^{3}$ and $33.5 \times 14.2 \times 4.9 \mathrm{~mm}^{3}$ are made along axial and circumferential directions, respectively (refer to Figures 5(c) and 5(d)).

The test set-up used for evaluation of performance of the GMR sensor array is shown in Figure 6. It consists of two saddle coils, variable DC power supply, track rope, flexible sensor array, GMR field meter, and a personal computer. Each saddle coil consists of 90 turns with a cross-sectional area of $20 \times 10 \mathrm{~mm}^{2}$. The centre-to-centre distance between the two saddle coils is $80 \mathrm{~mm}$. Measurements are made by moving the sensor array and the magnetization coils together as a single unit over the track rope. For this, a DC servo motor is used, as shown in Figure 6. In order to enhance the sensitivity, each GMR sensor output is amplified using low-noise differential amplifiers and notch rejection filter at $50 \mathrm{~Hz}$, followed by $100 \mathrm{kHz}$ low-pass filter. The sensor outputs are digitized using a 16-channel data acquisition system of 16-bit resolution.

The MFL signals of the sensor array for a circumferential LF of $5.5 \times 2.0 \times 2.0 \mathrm{~mm}^{3}$ in the track rope are shown in Figure 7. As the length of the flaw is $5.5 \mathrm{~mm}$, only two GMR sensors, namely, S6 and S7 have shown the output of the leakage flux.

The GMR array sensor output has been processed for removing background noise and formatted to obtain images. Typical MFL images of axial and circumferential LFs of size $5.5 \times 2.0 \times 2.0 \mathrm{~mm}^{3}$ are shown in Figures $8(\mathrm{a})$ and $8(\mathrm{~b})$, respectively. As compared to the MFL signals, it is possible to readily discern the spatial extent of the flaws from the MFL images produced by the sensor array. The MFL image of the axial LF is found to be extended as compared to that of the circumferential LF.

Typical MFL images of axial and circumferential LMAs are shown in Figures 9(a) and 9(b), respectively. As can be 


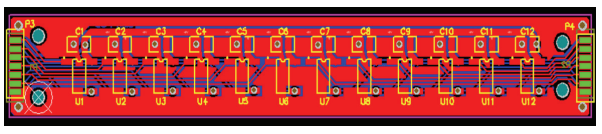

(a)

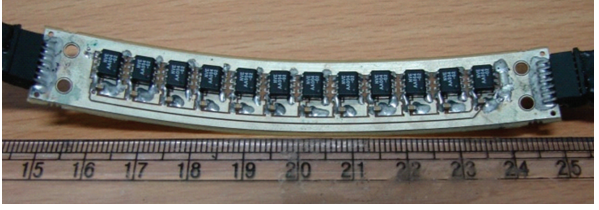

(b)

FIgURE 4: (a) the layout of the 12-element GMR sensor array on a flexible PCB and (b) the fabricated flexible sensor array.

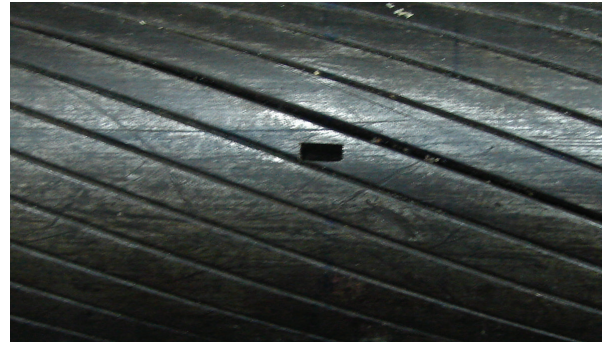

(a)

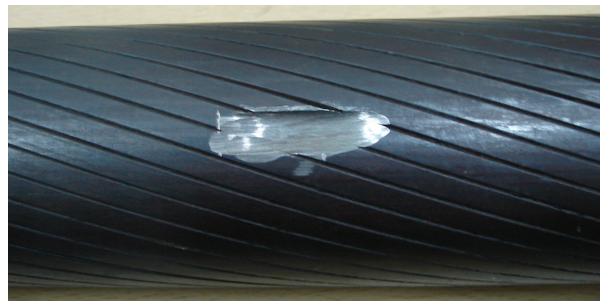

(c)

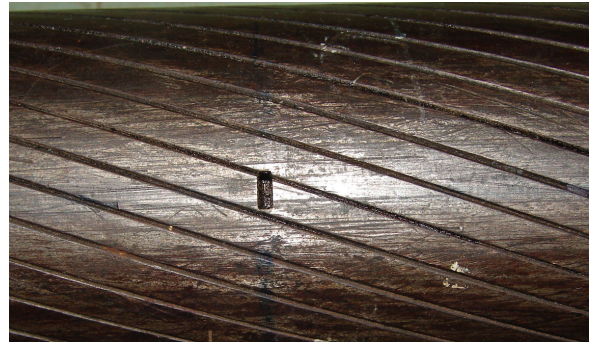

(b)

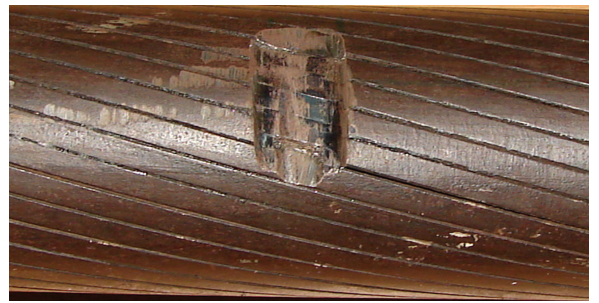

(d)

FIGURE 5: photographs of (a) axial LF, (b) circumferential LF, (c) axial LMA, and (d) circumferential LMA type defects in the track rope.

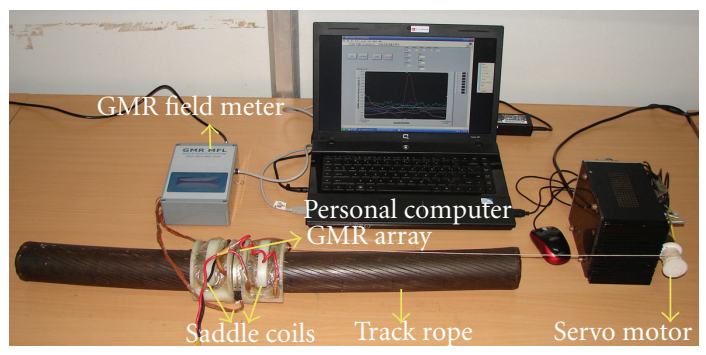

FIGURE 6: Test set-up used for performance evaluation of flexible GMR sensor array.

noted, the spatial extents of the LMAs could be readily felt from the images, despite some random noise. In the case of circumferential LMA, the output of three sensors, namely, S5, S6, and S7 that are exactly over the LMA defect have been found saturated due to high leakage field.

The flexible GMR sensor array designed has shown detection capability for both LF and LMA type defects oriented along the axial as well as circumferential directions. The sensor array has a fast detection speed along the length of the track rope and does not require circumferential scanning like in [13]. The images of circumferential notches have been found to be sharp and localized as compared to that of the

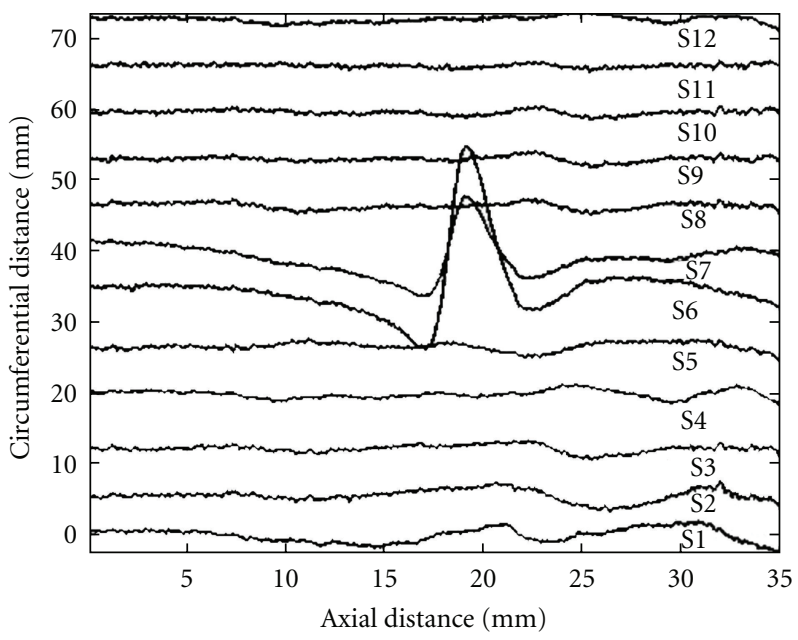

FIGURE 7: GMR sensor array response for a $5.5 \mathrm{~mm}$ long circumferential LF.

axial notches. Thus, the flexible GMR array sensor proposed in this paper can be used for rapid nondestructive inspection of track ropes. Towards deployment of the sensor array for field use, studies are in progress to assess the probability of detection (POD) of the MFL technique. Studies are also 


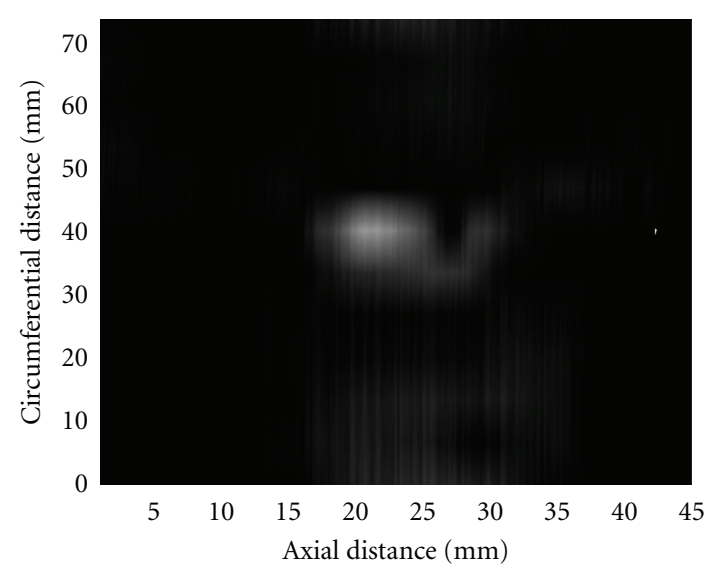

(a)

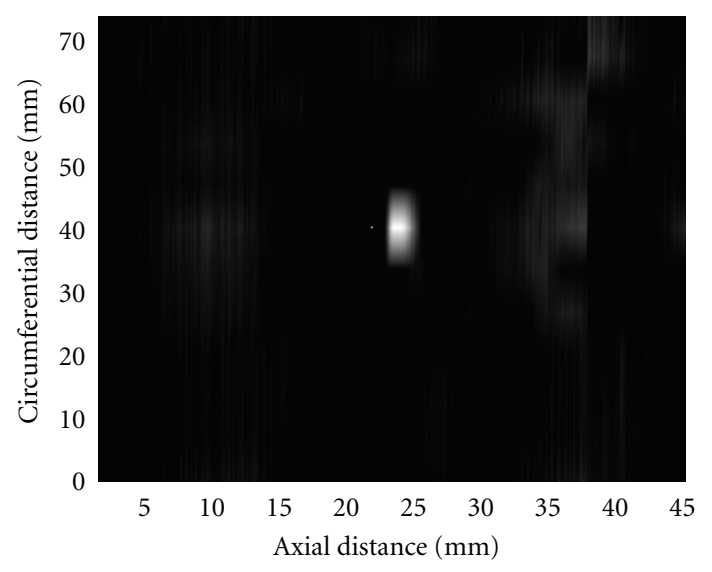

(b)

FigURE 8: MFL images for (a) axial and (b) circumferential LFs.

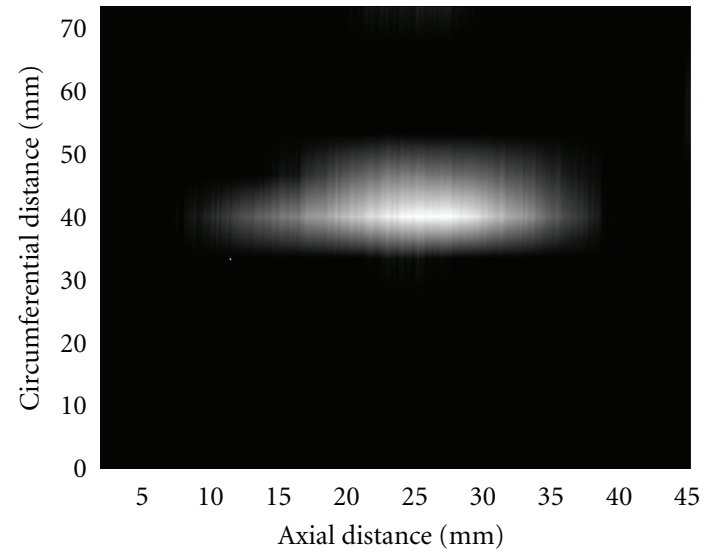

(a)

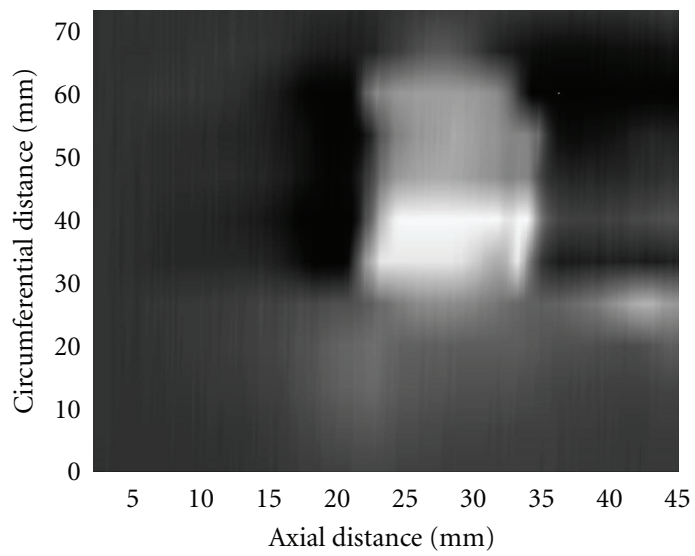

(b)

FIGURE 9: MFL images of (a) axial LMA $\left(42.0 \times 9.0 \times 3.0 \mathrm{~mm}^{3}\right)$ and (b) circumferential LMA $\left(33.5 \times 14.2 \times 4.9 \mathrm{~mm}^{3}\right)$.

in progress to enhance the detection capability for defects located in between sensor elements as well as the resolution of the sensor array, through the use of another tandem sensor array beside, with a slight angular shift.

\section{Summary}

A flexible GMR array sensor has been developed for fast magnetic flux leakage testing of $64 \mathrm{~mm}$ diameter steel track rope through detection of leakage magnetic fields from LF and LMA type defects on Z-wire rope. Three dimensional finite element modeling has been performed to identify the number of GMR sensor elements and their locations. The performance of the sensor array has been evaluated using machined LF and LMA type defects in the outer Z-wire. The flexible array sensor has shown detection of both types of defects in the track rope, with the possibility for imaging to obtain the spatial information of the defects.

\section{Acknowledgments}

The authors thank Mr. P. Krishnaiah, NDE Division, IGCAR, Kalpakkam for winding of the saddle coils and for his help during the experimental studies. They also thank Dr. C. K. Mukhopadhyay and Mr. S. Mahadevan for many useful discussions.

\section{References}

[1] D. Basak, "Performance comparison of drive ropes in a cable belt conveyor system using an NDT technique," Insight, vol. 51, no. 8, pp. 439-441, 2009.

[2] H. R. Weischedel and R. P. Ramsey, "Electromagnetic testing, a reliable method for the inspection of wire ropes in service," NDT International, vol. 22, no. 3, pp. 155-161, 1989.

[3] H. R. Weischedel, "The inspection of wire ropes in service: a critical review," Materials Evaluation, vol. 43, no. 13, pp. 1592$1605,1985$. 
[4] N. Sumyong, A. Prateepasen, and P. Kaewtrakulpong, "Influence of scanning velocity and gap distance on magnetic flux leakage measurement," ECTI Transactions on Electrical Engineering, Electronics and Communications, vol. 5, no. 1, pp. 118-122, 2007.

[5] C. Jomdecha and A. Prateepasen, "Design of modified electromagnetic main-flux for steel wire rope inspection," NDT and E International, vol. 42, no. 1, pp. 77-83, 2009.

[6] E. Kalwa and K. Piekarski, "Design of inductive sensors for magnetic testing of steel ropes," NDT International, vol. 20, no. 6, pp. 347-353, 1987.

[7] E. Kalwa and K. Piekarski, "Design of Hall-effect sensors for magnetic testing of steel ropes," NDT International, vol. 20, no. 5, pp. 295-301, 1987.

[8] W. S. Singh, B. P. C. Rao, S. Vaidyanathan, T. Jayakumar, and B. Raj, "Detection of leakage magnetic flux from near-side and far-side defects in carbon steel plates using a giant magnetoresistive sensor," Measurement Science and Technology, vol. 19, no. 1, Article ID 015702, 2008.

[9] J. W. Wilson and G. Y. Tian, "3D magnetic field sensing for magnetic flux leakage defect characterisation," Insight: NonDestructive Testing and Condition Monitoring, vol. 48, no. 6, pp. 357-359, 2006.

[10] W. S. Singh, B. P. C. Rao, T. Jayakumar, and B. Raj, "Simultaneous measurement of tangential and normal component of leakage magnetic flux using GMR sensors," Journal of NonDestructive Testing \& Evaluation, vol. 8, no. 2, pp. 23-28, 2009.

[11] A. Sophian, G. Y. Tian, and S. Zairi, "Pulsed magnetic flux leakage techniques for crack detection and characterisation," Sensors and Actuators, A, vol. 125, no. 2, pp. 186-191, 2006.

[12] J. W. Wilson and G. Y. Tian, "Pulsed electromagnetic methods for defect detection and characterisation," NDT and E International, vol. 40, no. 4, pp. 275-283, 2007.

[13] W. S. Singh, B. P. C. Rao, C. K. Mukhopadhyay, and T. Jayakumar, "GMR-based magnetic flux leakage technique for condition monitoring of steel track rope," Insight, vol. 53, no. 7, pp. 377-381, 2011.

[14] W. S. Singh, B. P. C. Rao, S. Mahadevan, and T. Jayakumar and B. Raj, "Giant magneto-resistive sensor based magnetic flux leakage technique for inspection of track ropes," Studies in Applied Electromagnetics and Mechanics, Electromagnetic Nondestructive Evaluation (XIV), vol. 35, pp. 256-263, 2011.

[15] R. Grimberg, L. Udpa, A. Savin, R. Steigmann, V. Palihovici, and S. S. Udpa, "2D Eddy current sensor array," NDT and E International, vol. 39, no. 4, pp. 264-271, 2006.

[16] M. N. Baibich, J. M. Broto, A. Fert et al., "Giant magnetoresistance of (001)Fe/(001)Cr magnetic superlattices," Physical Review Letters, vol. 61, no. 21, pp. 2472-2475, 1988.

[17] B. P. C. Rao, T. Jayakumar, and B. Raj, "Electromagnetic NDE techniques for materials characterization," in Ultrasonic and Advanced Methods for Non-Destructive Testing and Material Characterisation, C. H. Chen, Ed., chapter 11, pp. 262-265, World Scientific Publishing, Singapore, 2007. 

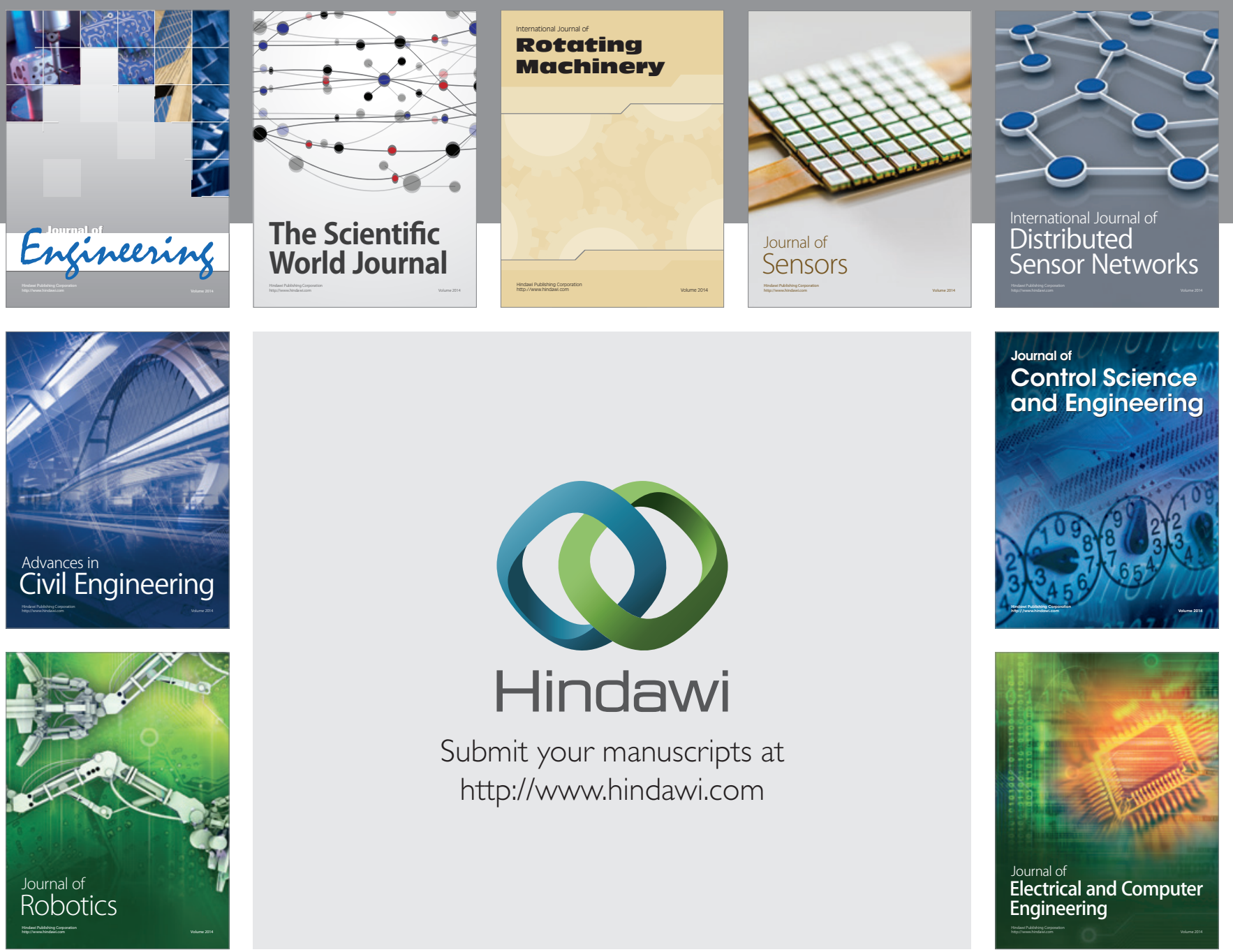

Submit your manuscripts at

http://www.hindawi.com
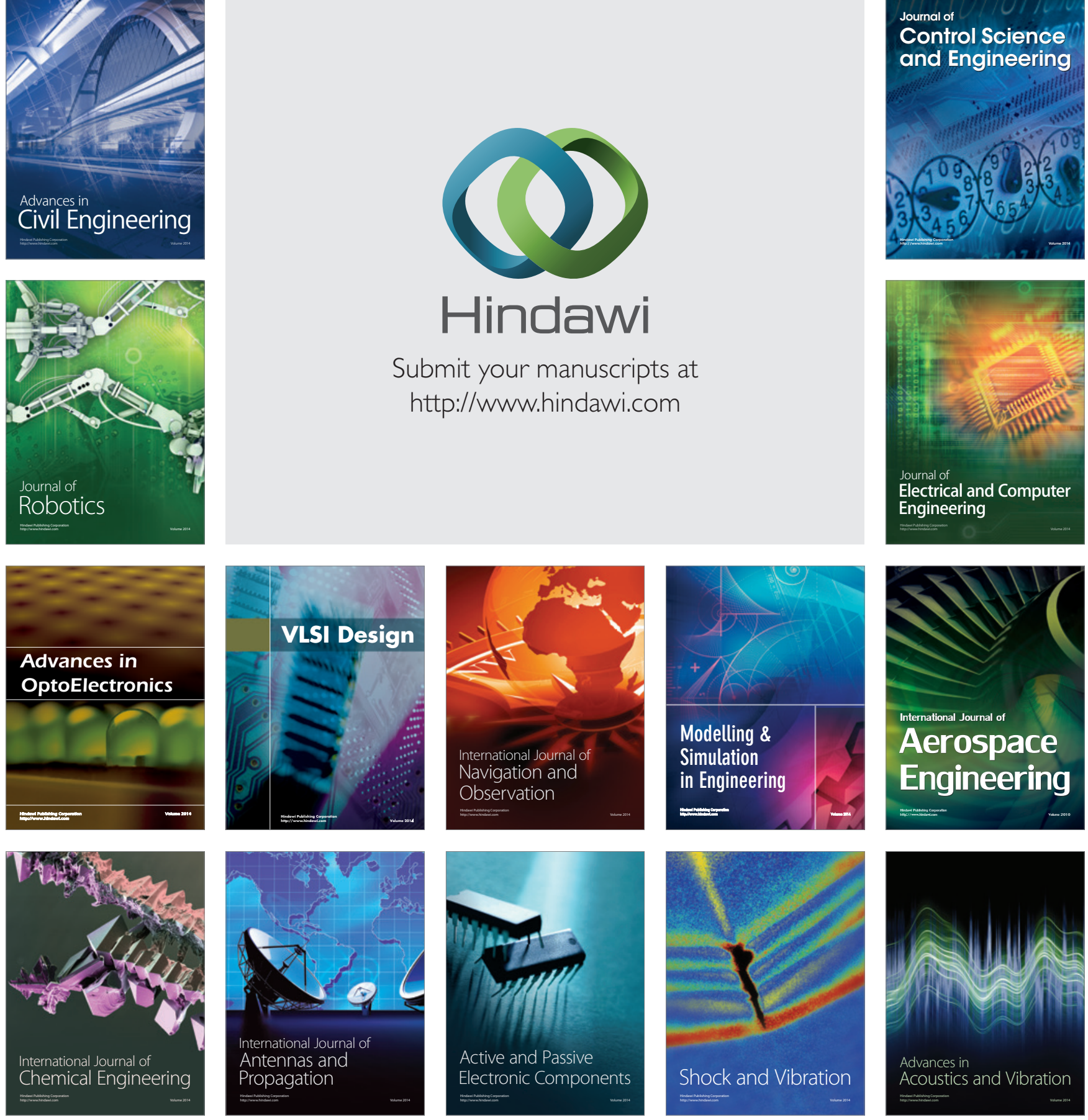